\title{
Mixed problem for quasilinear hyperbolic system with coefficients functionally dependent on solution
}

\author{
Małgorzata Zdanowicz and Zbigniew Peradzyński
}

\begin{abstract}
The mixed problem for quasilinear hyperbolic system with coefficients functionally dependent on the solution is studied. We assume that the coefficients are continuous nonlinear operators in the Banach space $C^{1}(\mathbb{R})$ satisfying some additional assumptions. Under these assumptions we prove the uniqueness and existence of local in time $C^{1}$ solution, provided that the initial data are also of class $C^{1}$.
\end{abstract}

\section{Introduction}

Since the beginning of 70-thies of the last century the Hall effect thrusters are more and more often used in the space technology not only for the correction of the satellites orbits but also as the marching engines in the space missions. Therefore one observes also a violent development of the theoretical studies of Hall thrusters. Although the physics laying behind the construction of such a thruster seems to be simple, there are still important problems and questions which are not yet solved. The rarified neutral gas, usually Xenon, moving through the chamber (a space between two concentric ceramical cylinders) is ionized by collisions with electrons. Neutral gas is released from appropriate

Key Words: Hyperbolic system, differential-functional equations, functional dependence on solution, method of characteristics, mixed problem.

2010 Mathematics Subject Classification: Primary 35L50, 39B, 65M25; Secondary 82D10.

Received: 04.11.2016

Revised: 15.12 .2016

Accepted: 21.12.2016 